\title{
The structure of 4-methylphenol and its water cluster revealed by rotationally resolved UV spectroscopy using a genetic algorithm approach
}

\author{
Grzegorz Myszkiewicz and W. Leo Meerts ${ }^{a)}$ \\ Molecular and Biophysics Group, Institute for Molecules and Materials, Radbound University Nijmegen, \\ P.O. Box 9010, NL-6500 GL Nijmegen, The Netherlands \\ Christian Ratzer and Michael Schmitt ${ }^{\text {b) }}$ \\ Institut für Physikalische Chemie, Heinrich-Heine-Universität 40225 Düsseldorf, Germany
}

(Received 24 March 2005; accepted 27 May 2005; published online 2 August 2005)

\begin{abstract}
The structure of 4-methylphenol ( $p$-cresol) and its binary water cluster have been elucidated by rotationally resolved laser-induced fluorescence spectroscopy. The electronic origins of the monomer and the cluster are split into four sub-bands by the internal rotation of the methyl group and of the hydroxy group in case of the monomer, and the water moiety in case of the cluster. From the rotational constants of the monomer the structure in the $S_{1}$ state could be determined to be distorted quinoidally. The structure of the $p$-cresol-water cluster is determined to be trans linear, with a O-O hydrogen bond length of $290 \mathrm{pm}$ in the electronic ground state and of $285 \mathrm{pm}$ in the electronically excited state. The $S_{1}$-state lifetime of $p$-cresol, $p$-cresol- $d_{1}$, and the binary water cluster have been determined to be 1.6, 9.7, and $3.8 \mathrm{~ns}$, respectively. (c) 2005 American Institute of Physics. [DOI: 10.1063/1.1961615]
\end{abstract}

\section{INTRODUCTION}

In the present study we present the determination of the geometry of $p$-cresol and its binary water cluster in the ground state and the electronically excited $S_{1}$ state by means of rotationally resolved electronic spectroscopy. The determination of excited state structures of aromatic molecules gives insight into the changes of the electronic properties of the chromophore. In a series of investigations, we determined the structural changes of some para-disubstituted aromatic molecules such as $p$-fluorophenol, ${ }^{1} p$-cyanophenol, ${ }^{2}$ and $p$-methylstyrene. ${ }^{3}$ In all these cases a quinoidal distortion along the $a$ axis of the molecule is found, whereas for singly substituted benzenes a symmetric distortion (benzenoid) of the aromatic ring is found. ${ }^{4}$ Changes of the electronic properties are also reflected in the change of the torsional barriers of symmetric (e.g., $\mathrm{CH}_{3}$ ) or asymmetric tops (e.g., $\mathrm{OH}$ ), which are directly attached to the benzene ring. In recent publications it was shown how the different electronic properties of the fluorine and the cyanogroup influence the barrier to hydroxy torsion in both electronic states of $p$-fluorophenol and $p$-cyanophenol. ${ }^{1,2}$

4-methylphenol possesses two feasible large amplitude motions: the methyl rotation with a mixed $V_{3} / V_{6}$ potential and the twofold hydroxy rotation. The molecular symmetry group that describes both motions is $G_{12}$. In the $p$-cresol-water cluster, the $\mathrm{OH}$ torsion is quenched; instead an additional splitting due to the torsional motion of the water moiety with respect to the water symmetry axis is observed. This barrier is expected from the similar phenol-

\footnotetext{
${ }^{a)}$ FAX: +33 24356 3311; electronic mail: leo.meerts@science.ru.nl

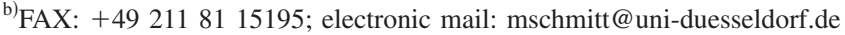

water system to be quite low. Thus, we have the case of twolow barrier torsional motions, which will split the origin into four sub-bands, an $A^{(3)}-E$ pair due to the threefold motion and an $A^{(2)}-B$ pair due to the twofold rotation. We use the superscripts (2) and (3) to distinguish the different $A$ sublevels from the two- and threefold internal rotation from the $A$ rotational constant. Since the rotational constants of this cluster are quite small, the overlapping of four subspectra leads to a very-congested overall spectrum in which it is difficult, if not impossible, to identify single rovibronic lines. However, the assignment of well-resolved lines to specific quantum numbers of the transitions is an indispensable prerequisite for an assigned fit of the molecular parameters. We have shown recently that in these cases of many overlapping lines, the use of a genetic algorithm approach for automated assignment of the spectra simplifies the interpretation and in many cases only makes it possible. ${ }^{5}$

4-methylphenol has found considerable interest as a chromophore and simple molecular model of the aromatic amino acid tyrosine. ${ }^{6}$ Proton transfer, electron transfer, and hydrogen transfer are important processes in clusters of tyrosine. E.g., in the water oxidizing complex of photosystem II, tyrosine is oxidized and reduced by hydrogen atom transfer involving tyrosine, water, and histidine in the active center. ${ }^{7}$

The electronic ground state of $p$-cresol has been investigated by infrared ${ }^{8,9}$ and Raman spectroscopy, ${ }^{6,10}$ by stimulated emission dip spectroscopy, ${ }^{11}$ and by dispersed fluorescence spectroscopy. ${ }^{12}$ Recently Lin et al. presented a massanalyzed threshold ionization spectrum of the $p$-cresol cation. $^{13}$ The nature of conformational preferences in $p$-cresol has been studied theoretically by Richardson et al. ${ }^{14}$ They determined the most stable conformation of $p$-cresol on 


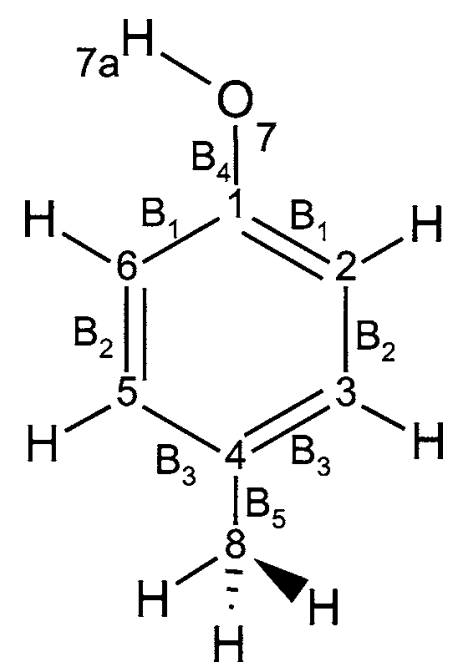

FIG. 1. The most stable conformation and atomic numbering of $p$-cresol.

MP2 level of theory to be eclipsed with respect to the methyl-group orientation, and the $\mathrm{OH}$ group on the same side of the aromatic ring as the eclipsed methyl hydrogen $(s y n)$, c.f. Fig. 1.

Several $p$-cresol-water clusters with up to four water molecules have been studied using dispersed fluorescence and- resonant two-photon ionization (R2PI) spectroscopy in the region of the intermolecular vibrations. ${ }^{15,16}$ From the vibrational analysis it is deduced that the binary cluster is hydrogen bound via a trans-linear $\mathrm{H}$ bond as in the similar phenol-water system. ${ }^{17}$

\section{EXPERIMENTAL SETUP}

The experimental setup for high-resolution laser-induced fluorescence (LIF) is described in detail elsewhere. ${ }^{18}$ Briefly, the molecular-beam machine consists of three differentially pumped vacuum chambers that are linearly connected by two skimmers with orifice diameters of $1 \mathrm{~mm}$ (first chamber) and $2 \mathrm{~mm}$ (second chamber) for the reduction of the Doppler width to $25 \mathrm{MHz}$. The expansion chamber is evacuated by a $8000-l / \mathrm{s}$ oil diffusion pump (Leybold DI 8000), which is backed by a $250-\mathrm{m}^{3} / \mathrm{h}$ roots blower pump (Saskia RPS 250) and a $65-\mathrm{m}^{3} / \mathrm{h}$ rotary pump (Leybold D65B). The second chamber serves as a buffer chamber and is pumped by a 400- $l / \mathrm{s}$ turbo-molecular pump (Leybold Turbovac 361), backed by a $40-\mathrm{m}^{3} / \mathrm{h}$ rotary pump (Leybold $\mathrm{D} 40 \mathrm{~B}$ ), maintaining a chamber pressure below $1 \times 10^{-5}$ mbar. The third chamber is pumped by a $145-l / \mathrm{s}$ turbo-molecular pump (Leybold Turbovac 151) through a liquid-nitrogen trap and backed by a $16-\mathrm{m}^{3} / \mathrm{h}$ rotary pump (Leybold D16B) resulting in a vacuum better than $1 \times 10^{-6}$ mbar. The molecular beam is crossed at right angles with the laser beam $360 \mathrm{~mm}$ downstream from the nozzle.

The laser system consists of a ring dye laser (Coherent 899-21), which is pumped with $6 \mathrm{~W}$ of the 514-nm line of an $\mathrm{Ar}^{+}$-ion laser (Coherent Innova 100). This light is coupled into an external-folded ring cavity (Spectra Physics) for second-harmonic generation (SHG). Typical UV powers are $10-40 \mathrm{~mW}$. The laser-induced fluorescence is collected perpendicular to the plane defined by the laser and molecular beam by an imaging optics setup consisting of a concave mirror and two plano-convex lenses. Data acquisition is performed and synchronized by means of a home-built real time LINUX program. ${ }^{19}$

\section{THEORY OF THE INTERNAL ROTATION}

The coupling between the internal rotation of the methyl group and the overall rotation of the molecule and the coupling of the $\mathrm{OH}$ and the water torsion with the overall rotation are treated in the formalism of the principal axis method (PAM). ${ }^{20,21}$ This is exact in the first (symmetric-top) case, but only an approximation for the twofold (asymmetric) top. Nevertheless as the hydrogen atom of the $\mathrm{OH}$ group and in the water moiety is light, and the inertial axes remain virtually unchanged during rotation of the top, the application of the PAM leads to reliable results. The Hamiltonian for the rotation-torsion interaction can be written as

$$
\hat{H}=\hat{H}_{R}+\hat{H}_{T}+\hat{H}_{\mathrm{RT}}
$$

with the rigid rotational Hamiltonian given by

$$
\hat{H}_{R}=A J_{a}^{2}+B J_{a}^{2}+C J_{c}^{2} .
$$

The torsional Hamiltonian for the mixed $V_{3} / V_{6}$ potential (methyl torsion) is given by

$$
\hat{H}_{T}=F p^{2}+\frac{1}{2} \sum_{n=1}^{2} V_{3 n}(1-\cos 3 n \alpha),
$$

and for a twofold $V_{2}$ potential ( $\mathrm{OH}$ and water torsion) by

$$
\hat{H}_{T}=F \rho^{2}+\frac{1}{2} \sum_{n=1}^{2} V_{2 n}(1-\cos 2 n \alpha) .
$$

$F$ is the torsional constant and defined by

$$
F=\frac{h}{8 \pi^{2} r I_{\alpha}}
$$

with

$$
r=1-\sum_{g=a, b, c} \frac{\lambda_{g}^{2} I_{\alpha}}{I_{g}} .
$$

Here the $\lambda_{g}(g=a, b, c)$ are the direction cosines between the inertial axes and the axis of internal rotation. $I_{\alpha}$ is the moment of inertia of the internal rotor with respect to its symmetry axis and $I_{g}$ are the principal moments of inertia of the entire molecule. In what follows, the treatment for the $n=2$ and $n=3$ potentials is identical.

The coupling of internal and overall rotation is described by $\hat{H}_{\mathrm{RT}}: 20,22$

$$
\begin{aligned}
\hat{H}_{\mathrm{RT}}= & F W_{v \sigma}^{(1)}\left(\rho_{a} J_{a}+\rho_{b} J_{b}+\rho_{c} J_{c}\right)+F W_{v \sigma}^{(2)}\left(\rho_{a} J_{a}+\rho_{b} J_{b}\right. \\
& \left.+\rho_{c} J_{c}\right)^{2},
\end{aligned}
$$

where the first-order perturbation coefficients $W_{\nu \sigma}^{(1)}$ are zero for the nondegenerate $A^{(2)}, A^{(3)}$, and $B$ levels. $W_{v \sigma}^{(2)}$ is nonzero for all levels. 
The coefficients $\rho_{g}$ with $g=a, b, c$ are defined as

$$
\begin{aligned}
& \rho_{a}=I_{\alpha} / I_{a} \cos \eta, \\
& \rho_{b}=I_{\alpha} / I_{b} \sin \eta,
\end{aligned}
$$

with $\eta$ as the angle between the projection vector of the $n$-fold rotor axis on the $a b$ plane and the $a$ axis.

The $n$ th-order perturbation coefficients $W_{v \sigma}^{(n)}$ in Eq. (7) are defined by ${ }^{20}$

$$
\begin{aligned}
& W_{v \sigma}^{(0)}=\frac{E_{v \sigma}}{F}, \\
& W_{v \sigma}^{(1)}=-2\langle v, \sigma|p| v, \sigma\rangle, \\
& W_{v \sigma}^{(2)}=1+4 F \sum_{v^{\prime}} \frac{\left|\left\langle v, \sigma|p| v^{\prime}, \sigma\right\rangle\right|^{2}}{E_{v \sigma}-E_{v^{\prime} \sigma}},
\end{aligned}
$$

where $|v, \sigma\rangle$ are eigenfunctions of Eqs. (3) and (4), and $E_{v \sigma}$ are the respective eigenvalues with $v$ as the torsional state index.

\section{THE GENETIC ALGORITHMS}

The experimental spectra were assigned automatically using a genetic algorithm-based fit described in detail in Refs. 5 and 23. We used the genetic algorithm (GA) library PGAPACK version 1.0, which can run on parallel processors. ${ }^{24}$ The calculations were performed on eight processors of a SUN UltraSPARC $333 \mathrm{MHz}$ and on a $2.6-\mathrm{GHz}$ personal computer (PC) with two processors under LINUX. The genetic algorithm uses concepts copied from natural reproduction and selection processes. For a detailed description of the GA the reader is referred to the original literature on evolutionary or genetic algorithms. ${ }^{25-27}$ In the present work a population of 300 was used and the calculation was completely converged after 500 generations. The other parameters which control the genetic algorithm convergency are similar as in Ref. 5.

The cost function used to describe the quality of the fit was defined as $C_{f g}=100\left(1-F_{f g}\right)$ with the fitness function $F_{f g}$ :

$$
F_{f g}=\frac{(\mathbf{f}, \mathbf{g})}{\|\mathbf{f}\|\|\mathbf{g}\|} .
$$

f and $\mathbf{g}$ represent the experimental and calculated spectra, respectively, and the inner product $(\mathbf{f}, \mathbf{g})$ is defined with the metric $\mathbf{W}$, which has the matrix elements $W_{i j}=w(|j-i|)$ $=w(r)$ as

$$
(\mathbf{f}, \mathbf{g})=\mathbf{f}^{T} \mathbf{W g},
$$

and the norm of $\mathbf{f}$ as $\|\mathbf{f}\|=\sqrt{(\mathbf{f}, \mathbf{f})}$ and similar for $\mathbf{g}$. For $w(r)$ we used a triangle function ${ }^{23}$ with a width of the base of $\Delta w$ :

$$
w(r)= \begin{cases}1-|r|\left(\frac{1}{2} \Delta w\right) & \text { for }|r| \leqslant \frac{1}{2} \Delta w \\ 0 & \text { otherwise. }\end{cases}
$$

Since the GA performs an intensity fit of the complete spectrum, much better information on the transition dipole moment (TDM) orientation and linewidth parameters are

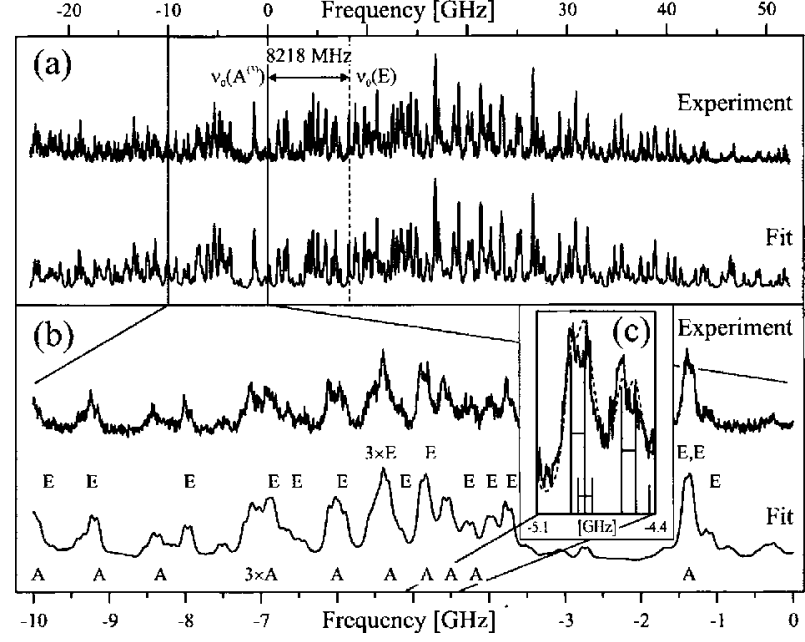

FIG. 2. (a) Experimental and fitted rovibronic spectrum of the electronic origin of 4-methylphenol. (b) $10-\mathrm{GHz}$ zoom of the (a) spectrum. (c) Inset showing three pairs of rovibronic transitions split by the twofold hydroxy group rotation in cresol. For the sake of clarity only the strongest transitions are shown and the splitting is marked with the horizontal lines. The solid line represents the experiment and the dashed line, the fit. All frequencies are given relative to the electronic origin at $35331.257(10) \mathrm{cm}^{-1}$.

gathered than from an intensity fit to a few individual lines. Thus, the GA results in improved values for the in plane angle $\theta$ of the TDM and also of the Lorentzian width of the individual rovibronic lines. Therefore, excited state lifetimes can be determined with a much higher accuracy than in lineshape fits of individual rovibronic lines.

\section{RESULTS AND DISCUSSION}

\section{A. 4-methylphenol}

Figure 2 shows the rotationally resolved LIF spectrum of the electronic origin of $p$-cresol. The spectrum is a pure $b$-type spectrum. It is split into two components, $A^{(3)}$ and $E$ by the internal rotation of the methyl group $\left(E_{v, 0}-E_{v, \pm 1}\right)$. Their respective origins, which are $8218 \mathrm{MHz}$ apart, are marked arrows.

In two recent publications ${ }^{3,28}$ we showed how a combined fit to high-resolution rovibronic data and lowresolution torsional transitions can be used to improve the determination of the barriers and torsional constants in both electronic states. $A, B, C, V_{3}, V_{6}, F$, and $\eta$ were fitted for each electronic state to the rotationally resolved origin and to the torsional transitions from Ref. 29. These torsional transitions are obtained from the zero-order perturbation coefficient, c.f. Eq. (9). The rovibronic spectrum was fitted using the genetic algorithm (GA) approach, described in Ref. 5. The parameters that determine the intensity and line form are the rotational temperatures, the Lorentzian and Gaussian linewidths, and the projections of the transition dipole moment on the inertial axes. They can be fitted most favorably using the GA algorithm. The Lorentzian contribution to the linewidth of the Voigt profile with a fixed Doppler width of $25 \mathrm{MHz}$ is determined to be $100 \pm 10 \mathrm{MHz}$, equivalent to a $S_{1}$-state lifetime of $1.6 \pm 0.2 \mathrm{~ns}$. The results of the fits are compiled in Tables I and II. 
TABLE I. Molecular parameters of 4-methylphenol and 4 -methyl $[7 D]$ phenol from the GA fit. The ground-state rotational constants of 4-methylphenol have been kept fixed at the microwave values from Ref. 30.

\begin{tabular}{|c|c|c|c|}
\hline 4-methylphenol & & $S_{0}$ & $S_{1}$ \\
\hline$A$ & $(\mathrm{MHz})$ & 5494.570 & $5154.6(12)$ \\
\hline$B$ & $(\mathrm{MHz})$ & 1456.963 & $1470.51(67)$ \\
\hline$C$ & $(\mathrm{MHz})$ & 1160.200 & $1153.62(41)$ \\
\hline$V_{3}$ & $(\mathrm{MHz})$ & $18.00(25)$ & $7.99(34)$ \\
\hline$V_{6}$ & $(\mathrm{MHz})$ & $-13.8(45)$ & $-24.7(43)$ \\
\hline$F$ & $\left(\mathrm{~cm}^{-1}\right)$ & $5.224(98)$ & $5.108(98)$ \\
\hline$\nu_{0}$ & $\left(\mathrm{~cm}^{-1}\right)$ & \multicolumn{2}{|c|}{$35331.257(10)$} \\
\hline$\nu_{0}(E)-\nu_{0}\left(A^{(3)}\right)$ & $(\mathrm{MHz})$ & \multicolumn{2}{|c|}{$8218(16)$} \\
\hline$\nu_{0}\left(A^{(2)}\right)-\nu_{0}(B)$ & $(\mathrm{MHz})$ & \multicolumn{2}{|c|}{$90(13)$} \\
\hline \multicolumn{4}{|c|}{ 4-methyl $[7 D]$ phenol } \\
\hline$A$ & $(\mathrm{MHz})$ & $5442.16(94)$ & $5110.04(94)$ \\
\hline$B$ & $(\mathrm{MHz})$ & $1417.59(37)$ & $1431.03(37)$ \\
\hline$C$ & $(\mathrm{MHz})$ & $1133.45(27)$ & $1127.46(27)$ \\
\hline$V_{3}$ & $(\mathrm{MHz})$ & $18.00(25)$ & $7.99(34)$ \\
\hline$V_{6}$ & $(\mathrm{MHz})$ & $-13.8(45)$ & $-24.7(43)$ \\
\hline$F$ & $\left(\mathrm{~cm}^{-1}\right)$ & $5.170(7)$ & $5.066(7)$ \\
\hline$\nu_{0}$ & $\left(\mathrm{~cm}^{-1}\right)$ & \multicolumn{2}{|c|}{$35326.729(10)$} \\
\hline$\nu_{0}(E)-\nu_{0}\left(A^{(3)}\right)$ & $(\mathrm{MHz})$ & \multicolumn{2}{|c|}{$8656.2(50)$} \\
\hline
\end{tabular}

Closer inspection of the spectrum shows that each of the rovibronic transitions is further split into two close lying sub-bands [c.f. Fig. 2(c)] due to the hindered $-\mathrm{OH}$ torsion. As a twofold torsion leads only to nondegenerate $A^{(2)}$ $(\sigma=0)$ and $B(\sigma=1)$ levels, the first-order perturbation coefficients from Eq. (10) are zero. The second-order perturbation coefficients which are nonzero [Eq. (11)] are quadratic in the angular momentum and can therefore be incorporated in the rotational constants. Since the $\mathrm{OH}$-torsional barrier is very high, the perturbations are small and both sub-bands can be fitted with the same set of effective rotational constants and an origin shift between the $A^{(2)}$ and $B$ bands of $90 \mathrm{MHz}$.

Determination of barriers and torsional constants in both electronic states demands more information from other spectroscopic techniques. In the microwave spectrum a splitting of the $b$-type transitions of $175 \mathrm{MHz}$ has been observed. ${ }^{30}$ The selection rule for $b$-type transitions is $\Delta \sigma= \pm 1$, so that the splitting between the subtorsional levels amounts to 87.5 MHz. The frequency of the pure torsional transition has

TABLE II. Torsional transitions of 4-methylphenol. The electronic transitions are labeled by the $m$ quantum number and the symmetry of the subtorsional level $\sigma: m \sigma\left(S_{1}\right) \leftarrow m \sigma\left(S_{0}\right)$ for absorption bands and $m \sigma\left(S_{1}\right)$ $\rightarrow m \sigma\left(S_{0}\right)$ for emission bands.

\begin{tabular}{ccccc}
\hline \hline$p$-cresol & Exp. $^{29}$ & Fit. & Diff. & \\
\hline $1 e \leftarrow 1 e$ & $8218(16)$ & 8214 & -4 & $\mathrm{MHz}$ \\
$2 e \leftarrow 1 e$ & $15.1(5)$ & 15.4 & +0.3 & $\mathrm{~cm}^{-1}$ \\
$3 a_{1} \leftarrow 0 a_{1}$ & $51.7(5)$ & 52.7 & +1.0 & $\mathrm{~cm}^{-1}$ \\
$4 e \leftarrow 1 e$ & $77(2)$ & 77.9 & +0.9 & $\mathrm{~cm}^{-1}$ \\
$5 e \leftarrow 1 e$ & $125(2)$ & 123.6 & -1.4 & $\mathrm{~cm}^{-1}$ \\
$1 e \rightarrow 2 e$ & $18.5(10)$ & 17.7 & -0.8 & $\mathrm{~cm}^{-1}$ \\
$0 a_{1} \rightarrow 3 a_{1}$ & $52.5(10)$ & 51.9 & -0.6 & $\mathrm{~cm}^{-1}$ \\
$1 e \rightarrow 4 e$ & $80.0(20)$ & 80.1 & +0.1 & $\mathrm{~cm}^{-1}$ \\
\hline \hline
\end{tabular}

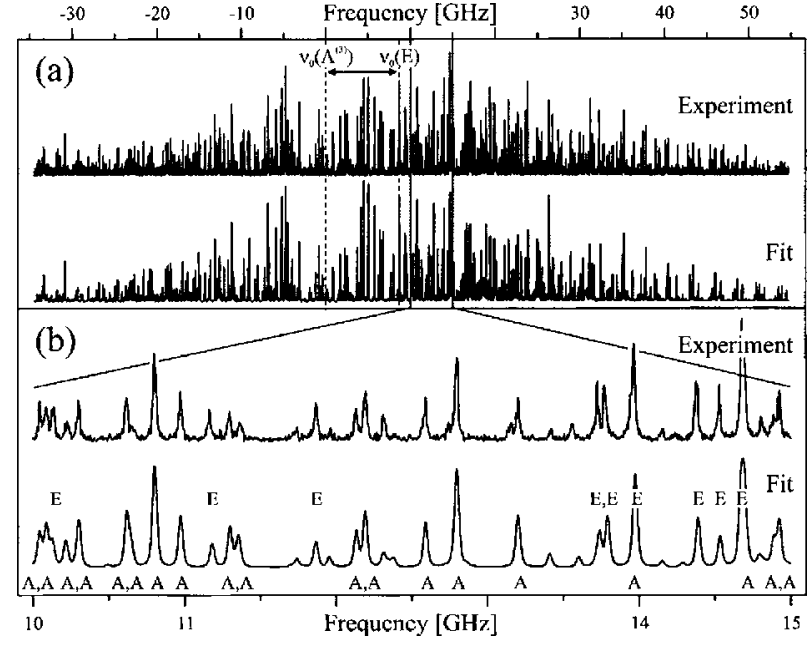

FIG. 3. Rovibronic spectrum of the electronic origin of 4-methyl $[7 D]$ phenol. Frequencies are given relative to the electronic origin at $35326.729(10) \mathrm{cm}^{-1}$.

been determined by IR spectroscopy to be $294 \mathrm{~cm}^{-1}$. 9 The first overtone of the torsional vibration in the electronically excited state is determined to be $1199 \mathrm{~cm}^{-1}$. Using these transitions and fixing the torsional constant $F$ at $690 \mathrm{GHz}$ (the value in phenol), we are able to fit the ground-state torsional $V_{2}$ barrier to $1130 \mathrm{~cm}^{-1}$ and the excited state barrier to $4395 \mathrm{~cm}^{-1}$. Both values are slightly lower than the corresponding values in phenol $\left(1215\right.$ and $4710 \mathrm{~cm}^{-1}$, respectively). ${ }^{17}$

\section{B. 4-methyl[7-D]phenol}

Figure 3 shows the rotationally resolved LIF spectrum of the electronic origin of 4-methyl[7-D]phenol. The spectrum is a pure $b$-type spectrum. Due to the heavier OD group, the twofold torsion is quenched in this system and the molecular symmetry group for the description of the molecule is $G_{6}$. Consequently, the origin band consists only of a split pair of $A^{(3)}$ and $E$ bands with a frequency spacing of $8656 \mathrm{MHz}$.

Table I gives the molecular parameters that have been determined using the GA program. The lifetime for 4-methyl $[7-D]$ phenol is determined to be $9.7 \pm 0.6 \mathrm{~ns}$ from a Lorentzian contribution to the total linewidth of $16.4 \pm 1 \mathrm{MHz}$. The $A^{(3)} E$ subtorsional splitting is very similar to the splitting in 4-methylphenol, also indicating similar potential barriers. As no torsional bands of 4-methyl $[7-D]$ phenol have been measured not enough data are available to fit the barriers to methyl rotation in this molecule. Nevertheless, since the effect of hydroxy deuteration on the methyl barrier is supposed to be small, we set barriers and torsional constants equal to those of 4-methylphenol.

\section{4-methylphenol $\left(\mathrm{H}_{2} \mathrm{O}\right)_{1}$}

Figure 4 presents the rotationally resolved electronic spectrum of the origin of the $p$-cresol-water cluster at $34972.873 \mathrm{~cm}^{-1}$. The origin is split into an $A^{(3)}$ and an $E$ sub-band due to the methyl torsion with a subtorsional splitting of $5459 \mathrm{MHz}$ and into an $A^{(2)}$ and $B$ sub-band due to the water torsion with a subtorsional splitting of $29428 \mathrm{MHz}$. 


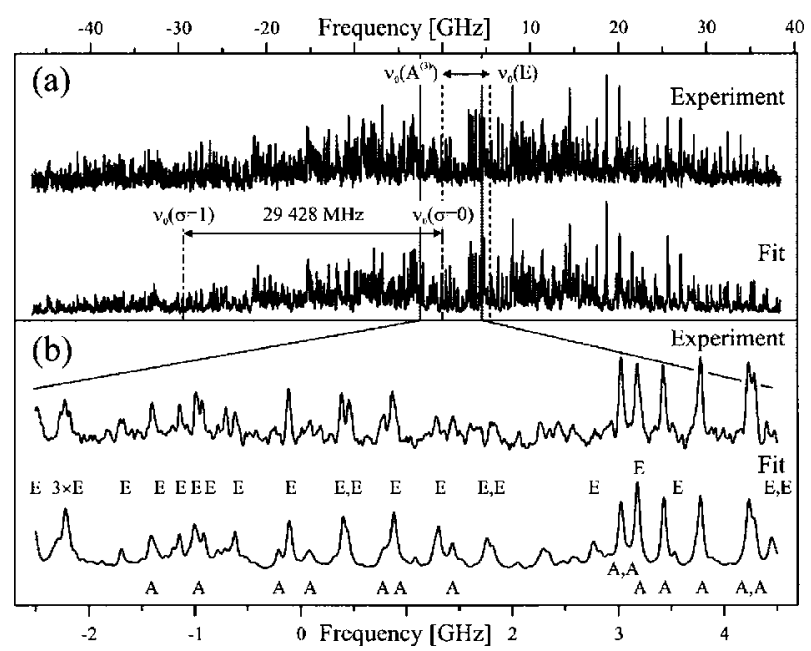

FIG. 4. Rovibronic spectrum of the electronic origin of 4-methylphenol water. Frequencies are given relative to the electronic origin at 34 972.873(10) $\mathrm{cm}^{-1}$.

The angle of the transition dipole moment with the $a$ axis is determined from the relative intensities of $a$ and $b$ bands to be $76.70^{\circ}$. The rotation of $13.3^{\circ}$ with respect to the monomer is in good agreement with a value of $20^{\circ}$, that is predicted by rotation of the inertial axis upon cluster formation on the basis of the ab initio structure.

As in the case of phenol, the subtorsional structure due to the twofold water torsion can be fit using a rigid-rotor model with different rotational constants for both components. Similar to the phenol-water case, the $B$ and $C$ rotational constants of the $(\sigma=0)$ and $(\sigma=1)$ components are equal within their uncertainties. The $A$ constants of the subtorsional components differ by about $11 \mathrm{MHz}$, indicating that the torsional axis of the water torsion is more or less parallel to the main inertial $a$ axis of the cluster. This structure can be described as a trans-linear hydrogen bond configuration, with the water moiety acting as proton acceptor, like in phenol water. A spin statistics of 1:3 for the ratio of $(\sigma=0)$ to $(\sigma=1)$ further supports a structure with the water moiety symmetric with respect to the aromatic plane.

This analysis clearly shows the convenience of the GA analysis of such a complex spectrum. Although by eye no periodicity can be observed at first glance, the GA is capable of easily finding the two splittings from the methyl torsion
(5459 MHz) and the water torsion (29 $428 \mathrm{MHz}$ ). Of course, the application of the GA-based-automated technique still requires the choice of the "correct" Hamiltonian for the problem under consideration. An easy way of finding spectral splittings (and thus obtaining information about the required Hamiltonian) is to perform an autocorrelation of the spectrum. This method has been shown by Helm et al. to be helpful in the analysis of the phenol-water cluster spectrum ${ }^{31}$ and later by Remmers et al. in the analysis of the tunneling spectrum of the benzoic acid dimer. ${ }^{32}$ Nevertheless, if one component of the complete spectrum amounts to more than $50 \%$ of the total intensity, the GA is capable of fitting this part of the spectrum, although it might be concealed in the dense spectrum of other component(s) and would therefore be inaccessible to a classical analysis using line-positionassigned fits. From the difference of the partly fitted spectrum to the experimental one, the missing components can easily be recognized and subsequently fitted.

In order to compute the geometrical rotational constants from the torsionally perturbed constants of Table III, we have to estimate the $V_{2}$ potential energy barrier and the torsional constant $F$ for the water torsion in both electronic states. However, only three pieces of information are available for the determination of these four parameters: the $(\sigma=0) /$ $(\sigma=1)$ subtorsional splitting of $29428 \mathrm{MHz}$, and the difference of the $A$ rotational constants for $(\sigma=0)$ and $(\sigma=1)$ in the electronic ground state and in the electronically excited state. Fixing $F^{\prime \prime}$ of the ground state to the value determined for phenol water ${ }^{28}\left(14.813 \mathrm{~cm}^{-1}\right)$ (the ground-state acidities of phenol and cresol are similar, therefore one would expect similar torsional constants for the water moiety), a groundstate barrier $V_{2}^{\prime \prime}$ of $182.6 \mathrm{~cm}^{-1}$, an excited state barrier $V_{2}^{\prime}$ of $125.3 \mathrm{~cm}^{-1}$, and a torsional constant $F^{\prime}$ in the excited state of $14.9 \mathrm{~cm}^{-1}$ are calculated. These values are very close to the torsional barriers of phenol water.

\section{Determination of the structures}

The program pKrFit (Ref. 4) was used to determine the structure of 4-methylphenol in the $S_{0}$ and $S_{1}$ states from the rotational constants of the two isotopomers described above. Due to the limited number of isotopomers in this study, we performed a fit to the $r_{0}$ structure, which completely neglects the vibrational contributions from the different isotopomers.

TABLE III. Molecular parameters of 4-methylphenol water.

\begin{tabular}{|c|c|c|c|c|c|}
\hline & & \multicolumn{2}{|c|}{$S_{0}$} & \multicolumn{2}{|c|}{$S_{1}$} \\
\hline & & $\sigma=0$ & $\sigma=1$ & $\sigma=0$ & $\sigma=1$ \\
\hline$A$ & $(\mathrm{MHz})$ & $3663.48(244)$ & $3652.19(53)$ & $3590.87(244)$ & $3576.37(53)$ \\
\hline$B$ & $(\mathrm{MHz})$ & $765.60(59)$ & $765.98(31)$ & 774.78(59) & $774.35(31)$ \\
\hline$C$ & $(\mathrm{MHz})$ & $637.00(48)$ & $637.71(31)$ & $640.76(48)$ & $641.61(31)$ \\
\hline$V_{3}$ & $(\mathrm{MHz})$ & \multicolumn{2}{|c|}{$18.00(25)$} & \multicolumn{2}{|c|}{$7.99(34)$} \\
\hline$V_{6}$ & $(\mathrm{MHz})$ & \multicolumn{2}{|c|}{$-13.8(45)$} & \multicolumn{2}{|c|}{$-24.7(43)$} \\
\hline$F$ & $\left(\mathrm{~cm}^{-1}\right)$ & \multicolumn{2}{|c|}{$5.41(7)$} & \multicolumn{2}{|c|}{$5.21(7)$} \\
\hline$\eta$ & $\left(^{\circ}\right)$ & \multicolumn{2}{|c|}{ 19.1(10) } & \multicolumn{2}{|c|}{ 19.1(10) } \\
\hline$\nu_{0}$ & $\left(\mathrm{~cm}^{-1}\right)$ & & & $34971.891(10)$ & $34972.873(10)$ \\
\hline$\nu_{0}\left(A^{(2)}\right)-\nu_{0}(B)$ & $(\mathrm{MHz})$ & \multicolumn{4}{|c|}{$29428(7)$} \\
\hline$\nu_{0}\left(A^{(3)}\right)-\nu_{0}(E)$ & $(\mathrm{MHz})$ & \multicolumn{4}{|c|}{$5459(6)$} \\
\hline
\end{tabular}


TABLE IV. Structural $r_{0}$ parameters of $p$-cresol. For atomic numbering see Fig. 1. All values are in pm.

\begin{tabular}{ccr}
\hline \hline & $B_{1}\left(C_{1} C_{2}\right)$ & $139.9(1)$ \\
& $B_{2}\left(C_{2} C_{3}\right)$ & $140.5(3)$ \\
$S_{0}$ & $B_{3}\left(C_{3} C_{4}\right)$ & $138.9(1)$ \\
& $B_{4}(\mathrm{CO})$ & $138.3(6)$ \\
& $B_{5}\left(\mathrm{C} C_{\text {methyl }}\right)$ & $151.3(6)$ \\
& & \\
& $B_{1}\left(C_{1} C_{2}\right)$ & $142.2(10)$ \\
& $B_{2}\left(C_{2} C_{3}\right)$ & $138.1(10)$ \\
$S_{1}$ & $B_{3}\left(C_{3} C_{4}\right)$ & $144.3(4)$ \\
& $B_{4}(\mathrm{CO})$ & $134.5(11)$ \\
& $B_{5}\left(\mathrm{CC} C_{\text {methyl }}\right)$ & $150.3(2)$ \\
\hline \hline
\end{tabular}

A simple model for the geometry has been adopted, that is given in Fig. 1. All aromatic $\mathrm{CH}$ bonds are set to the same value $(107.7 \mathrm{pm}$ for the ground state and $107.4 \mathrm{pm}$ for the excited state), the $\mathrm{CH}$ bonds of the methyl group are fixed at $109.0 \mathrm{pm}$. The $\mathrm{OH}$ bond lengths from the structural fits of phenol have been taken as constants $\left(96 \mathrm{pm}\right.$ in the $S_{0}$ and $99 \mathrm{pm}$ in the $S_{1}$ states). Opposing CC bonds are set equal.

Table IV gives the results for the fits of the $S_{0}$ and the $S_{1}$ structure. The aromatic ring expands upon electronic excitation quinoidally, as has been found for other paradisubstituted aromatic compounds. The $\mathrm{CO}$ and the $\mathrm{CC}_{\text {methyl }}$ bond lengths decrease, which are in agreement with a quinoidal distortion of the ring system. The relative shortening of these bands cannot be determined accurately, as both bonds nearly coincide with the main inertial $a$ axis. Because the deuteration is at a position close to this axis, both bond lengths are strongly correlated. The results present therefore only one possible combination of the bond length decreases.

From the above determined $S_{0}$ and $S_{1}$ structures of the cresol moiety, we fitted the structure of the $p$-cresol water to the geometric rotational constants. All geometry parameters in $p$-cresol have been kept fixed at the monomer values, the geometry of the water moiety has also been kept fixed. We imposed the symmetry constraint on the fit: The $\mathrm{H}$ atoms of the water moiety are symmetric with respect to the aromatic plane. Table $\mathrm{V}$ shows the results.

The $\mathrm{O}-\mathrm{O}$ hydrogen bond length decreases by $5.0 \mathrm{pm}$ upon electronic excitation, imaging the increased acidity of $p$-cresol upon electronic excitation, while the OOC angle and the HOOC dihedral, describing the orientation of the water

TABLE V. Structural $r_{0}$ parameters of $p$-cresol water. The atomic numbering refers to Fig. 1, the subscript $w$ refers to atoms of the water moiety. The dihedral angle is defined between the $\mathrm{H}_{1}$ atom of the water moiety, the $\mathrm{O}$ atom of water, the $\mathrm{O}_{7}$ atom of cresol, and the $\mathrm{C}_{1}$ atom of cresol. The second dihedral angle is determined by the symmetry constraint.

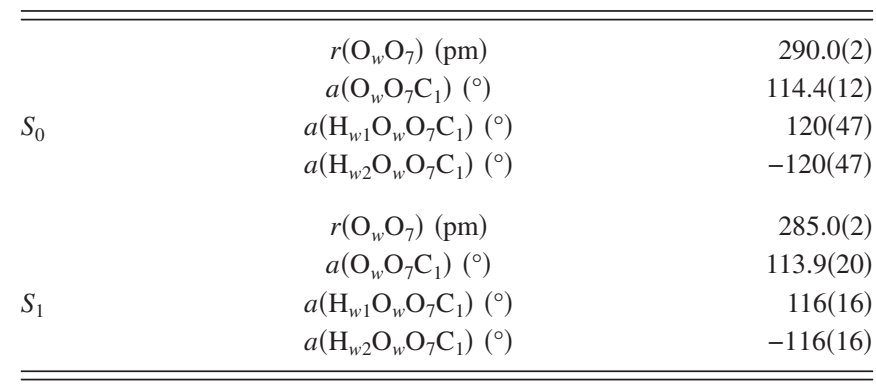

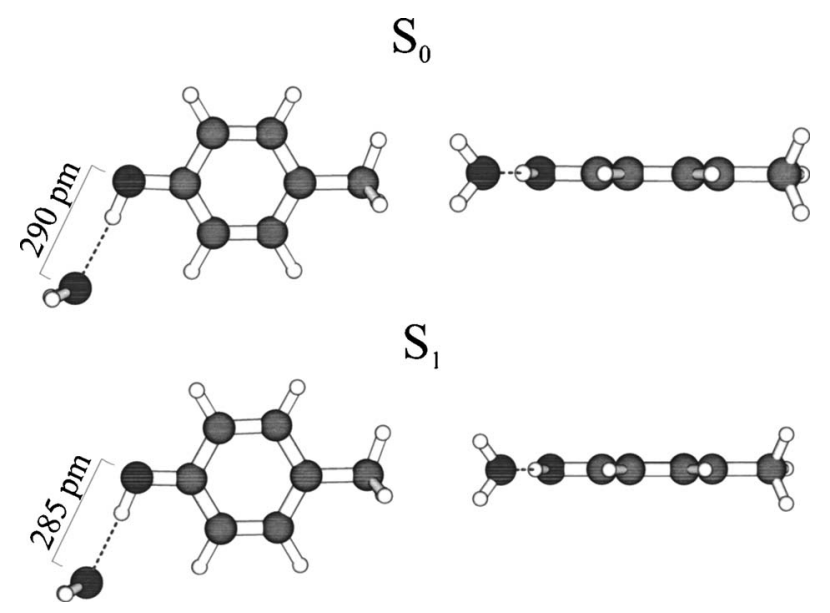

FIG. 5. Top and side views of the ground and excited state structures of $p$-cresol water. For all other geometry parameters see Table V.

moiety, nearly stay constant. Two different views of the ground and excited state structure are shown in Fig. 5.

\section{CONCLUSIONS}

The value for the torsional barrier due to the internal rotation of the hydroxy group in $p$-cresol was found to be $1130 \mathrm{~cm}^{-1}$ in the ground state and $4395 \mathrm{~cm}^{-1}$ in the excited state. These values can be compared to the $V_{2}$ barriers of other $p$-disubstituted phenols and of phenol itself, given in Table VI.

Relative to phenol, para substitution with fluorine (+mesomeric effect) and methyl (+inductive effect) leads to a decrease of the torsional barrier in both electronic states, while the cyano group (-mesomeric effect) increases the barrier. Electron accepting groups (as cyano) stabilize a partial quinoidal structure of the $p$-substituted phenol with some double bond characters of the $\mathrm{CO}$ bond, while electron donating groups destabilize it. The lifetime of 4-methyl $[7-D]$ phenol determined from the Lorentz contribution to the Voigt profile $(9.7 \mathrm{~ns})$ is substantially longer than the respective 4-methylphenol lifetime of $1.6 \mathrm{~ns}$. A similar increase of the $S_{1}$-state lifetime was found for phenol and $[7 D]$ phenol and was explained by a smaller probability for tunneling of the OD species through the barrier which separates the ${ }^{1} \pi \sigma^{*}$ from the ${ }^{1} \pi \pi^{*}$ surface. ${ }^{33}$

The $V_{3}$ and $V_{6}$ barriers of the methyl-group torsion are low, as is typical for molecules with $G_{12}$ symmetry. Barriers determined from 4-methylphenol and 4-methyl[7-D]phenol are equal within the uncertainty, showing that the two torsional motions are mainly decoupled.

TABLE VI. OH-torsional barriers of phenol, $p$-fluorophenol, $p$-cyanophenol, and $p$-methylphenol ( $p$-cresol). All values are in $\mathrm{cm}^{-1}$.

\begin{tabular}{lccc}
\hline \hline & \multicolumn{2}{c}{$V_{2}$ barrier } & \\
\cline { 2 - 3 } & $S_{0}$ & $S_{1}$ & References \\
\hline$p$-fluorophenol & 1006 & 1819 & 1 \\
$p$-methylphenol & 1130 & 4395 & This work \\
phenol & 1215 & 4710 & 17 \\
$p$-cyanophenol & 1420 & $>5000$ & 2 \\
\hline
\end{tabular}


From the rotational constants of both isotopomers, the structural change upon electronic excitation could be determined. As it is typical for para-disubstituted aromatics, the ring expands quinoidally upon excitation, while the two bonds in the para position decrease. The decrease of the $\mathrm{CO}$ bond length mirrors the shift of electron density from the oxygen atom to the aromatic ring, which takes place and is the reason for the increased acidity of phenols upon electronic excitation.

The rotationally resolved electronic spectrum of the origin of the water cluster is split into an $A^{(3)} E$ pair due to the methyl-group rotation and into an $A^{(2)} B$ pair due to the torsional motion of the water moiety. The $S_{1}$ lifetime of the $p$-cresol-water cluster determined from the Lorentz contribution to the Voigt profile is $42 \pm 5 \mathrm{MHz}$, equivalent to a $S_{1}$-state lifetime of $3.8 \pm 0.5 \mathrm{~ns}$, which is much shorter than the lifetime of the similar phenol-water cluster (15 ns). The large increase of the lifetime by going from phenol to the phenol-water cluster was attributed by Sobolewski and Domcke to a removal of the conical intersection of the ${ }^{1} \pi \sigma^{*}$ surface with the ground state. Furthermore, the ${ }^{1} \pi \sigma^{*}$ surface shifts to higher energies, and develops a minimum at the hydrogen-transferred geometry of the cluster. ${ }^{33}$ The longer lifetimes of deuterated phenol and the phenol-water cluster compared to phenol have therefore different explanations (smaller tunneling rate versus removal of a conical intersection). For the explanation of the lifetimes, it was also discussed that a rapid internal conversion takes place with the $\mathrm{OH}$ stretching vibration as an accepting mode. ${ }^{34,35}$ Both deuteration and complexation with water lower the stretching frequency of the $\mathrm{OH}$ vibration, reducing its ability to act as an accepting mode. In this picture the lifetime of the $p$-cresol-water cluster should be equally increased as the one of deuterated $p$-cresol. However, we found a much shorter lifetime for the cresol-water cluster than for the deuterated cresol, strongly favoring the lifetime model of Sobolewski and Domcke. ${ }^{33}$

The structure of the $p$-cresol-water cluster could be determined to be trans-linearly hydrogen bound, with cresol as proton donor like in the similar phenol-water cluster. The $\mathrm{OO}$ hydrogen bond length could be determined to be $290 \mathrm{pm}$ in the ground state and to $285 \mathrm{pm}$ in the electronically excited state. This decrease in hydrogen bond length is a consequence of the increased acidity of $p$-cresol in the $S_{1}$ state, which leads to a stronger hydrogen bond. Again, the decrease of the hydrogen bond length of $5 \mathrm{pm}$ is very similar to the corresponding value in phenol water $(4 \mathrm{pm})$.

\section{ACKNOWLEDGMENTS}

The financial support of the Deutsche Forschungsgemeinschaft (SCHM 1043/9-3) is gratefully acknowledged.
One of the authors (M.S.) would like to thank the Nordrheinwestfälische Akademie der Wissenschaften for a grant which made this work possible.

${ }^{1}$ C. Ratzer, M. Nispel, and M. Schmitt, Phys. Chem. Chem. Phys. 5, 812 (2002)

${ }^{2}$ J. Küpper, M. Schmitt, and K. Kleinermanns, Phys. Chem. Chem. Phys. 4, 4634 (2002).

${ }^{3}$ M. Schmitt, C. Ratzer, C. Jacoby, and W. L. Meerts, J. Mol. Struct. 742, 123 (2005).

${ }^{4}$ C. Ratzer, J. Küpper, D. Spangenberg, and M. Schmitt, Chem. Phys. 283, 153 (2002).

${ }^{5}$ W. L. Meerts, M. Schmitt, and G. Groenenboom, Can. J. Chem. 82, 804 (2004).

${ }^{6}$ Z. Arp, D. Autrey, J. Laane, S. A. Overman, and G. J. Thomas, Biochemistry 40, 2522 (2001).

${ }^{7}$ M. Blomberg and P. Siegbahn, Mol. Phys. 101, 323 (2001).

${ }^{8} \mathrm{G}$. Varsanyi, Assignments for Vibrational Spectra of 700 Benzene Derivatives (Wiley, New York, 1974).

${ }^{9}$ R. J. Jacobsen, Spectrochim. Acta 21, 433 (1965).

${ }^{10}$ J. Laane, K. Haller, S. Sakurai, K. Morris, D. Autrey, Z. Arp, W. Chiang, and A. Combs, J. Mol. Struct. 650, 57 (2003).

${ }^{11}$ T. Ebata and M. Ito, J. Phys. Chem. 96, 3224 (1992).

${ }^{12}$ K. Song and J. M. Hayes, J. Mol. Spectrosc. 134, 82 (1989).

${ }^{13}$ J. L. Lin, C. Li, and W. B. Tzeng, J. Chem. Phys. 120, 10513 (2004).

${ }^{14}$ P. R. Richardson, M. A. Chapman, D. C. Wilson, S. P. Bates, and A. C. Jones, J. Chem. Phys. 4, 4910 (2002).

${ }^{15}$ M. Pohl, M. Schmitt, K. Wolf, and K. Kleinermanns, J. Chem. Phys. 94, 1717 (1991).

${ }^{16}$ M. Pohl, M. Schmitt, and K. Kleinermanns, Chem. Phys. Lett. 177, 252 (1991).

${ }^{17}$ G. Berden, W. L. Meerts, M. Schmitt, and K. Kleinermanns, J. Chem. Phys. 104, 972 (1996).

${ }^{18}$ M. Schmitt, J. Küpper, D. Spangenberg, and A. Westphal, Chem. Phys. 254, 349 (2000).

${ }^{19}$ J. Küpper, Ph.D. thesis, Heinrich-Heine-Universität, Düsseldorf, 2000.

${ }^{20}$ W. Gordy and R. L. Cook, Microwave Molecular Spectra, 3rd ed. (Wiley, New York, 1984).

${ }^{21}$ C. C. Lin and J. D. Swalen, Rev. Mod. Phys. 31, 841 (1959).

${ }^{22}$ D. R. Herschbach, J. Chem. Phys. 31, 91 (1959).

${ }^{23}$ J. A. Hageman, R. Wehrens, R. de Gelder, W. L. Meerts, and L. M. C. Buydens, J. Chem. Phys. 113, 7955 (2000).

${ }^{24}$ D. Levine, PGAPACK V1.0, PGAPACK can be obtained via anonymous ftp from ftp://ftp.mcs.anl.gov/pub/pgapack/ pgapack.tar.z., 1996.

${ }^{25}$ J. H. Holland, Adaption in Natural and Artificial Systems (University of Michigan Press, Ann-Arbor, MI, 1975).

${ }^{26}$ D. E. Goldberg, Genetic Algorithms in Search, Optimisation and Machine Learning (Addison-Wesley, Reading, Massachusetts, 1989).

${ }^{27}$ I. Rechenberg, Evolutionsstrategie-Optimierung Technischer Systeme Nach Prinzipien der Biologischen Evolution (Frommann-Holzboog, Stuttgart, 1973).

${ }^{28}$ C. Jacoby and M. Schmitt, ChemPhysChem 5, 1686 (2004).

${ }^{29}$ M. Schmitt, Ph.D. thesis, Ruprecht-Karl-Universität, Heidelberg, 1992.

${ }^{30} \mathrm{~A}$. Hellweg, in Mikrowellenspektroskopische Untersuchungen zur intramolekularen Dynamik von p-Toluidin, p-Kresol und p-Thiokresol, edited by Günter Mainz (Verlag Gýnter Mainz, Aachen, 2003).

${ }^{31}$ R. M. Helm, H. P. Vogel, and H. J. Neusser, J. Chem. Phys. 108, 4496 (1998).

${ }^{32}$ K. Remmers, W. L. Meerts, and I. Ozier, J. Chem. Phys. 112, 10890 (2000).

${ }^{33}$ A. L. Sobolewski and W. Domcke, J. Phys. Chem. A 105, 9275 (2001).

${ }^{34}$ A. Sur and P. M. Johnson, J. Chem. Phys. 84, 1206 (1986).

${ }^{35}$ R. J. Lipert and S. D. Colson, J. Phys. Chem. 93, 135 (1989). 